PROCEEDINGS OF THE

AMERICAN MATHEMATICAL SOCIETY

Volume 134, Number 11, November 2006, Pages 3329-3341

S 0002-9939(06)08602-3

Article electronically published on May 12, 2006

\title{
A CLASS OF INTEGRAL IDENTITIES WITH HERMITIAN MATRIX ARGUMENT
}

\author{
DAYA K. NAGAR, ARJUN K. GUPTA, AND LUZ ESTELA SÁNCHEZ
}

(Communicated by Richard A. Davis)

\begin{abstract}
The gamma, beta and Dirichlet functions have been generalized in several ways by Ingham, Siegel, Bellman and Olkin. These authors defined them as integrals having the integrand as a scalar function of real symmetric matrix. In this article, we have defined and studied these functions when the integrand is a scalar function of Hermitian matrix.
\end{abstract}

\section{INTRODUCTION}

Several generalizations of Euler's gamma function are available in the scientific literature. The multivariate gamma function which is frequently used in multivariate statistical analysis is defined by (Ingham [4, Siegel [10])

$$
\Gamma_{m}(a)=\int_{X>0} \operatorname{etr}(-X) \operatorname{det}(X)^{a-(m+1) / 2} d X,
$$

where $\operatorname{etr}(A)=\exp (\operatorname{tr}(A))$, $\operatorname{det}(A)=$ determinant of $A$, and the integration is carried out over $m \times m$ symmetric positive definite matrices. By evaluating the above integral it is easy to see that

$$
\Gamma_{m}(a)=\pi^{m(m-1) / 4} \prod_{j=1}^{m} \Gamma\left(a-\frac{j-1}{2}\right), \operatorname{Re}(a)>\frac{m-1}{2} .
$$

The multivariate generalization of the beta function is given by

$$
\begin{aligned}
B_{m}(a, b) & =\int_{0<X<I_{m}} \operatorname{det}(X)^{a-(m+1) / 2} \operatorname{det}\left(I_{m}-X\right)^{b-(m+1) / 2} d X \\
& =\frac{\Gamma_{m}(a) \Gamma_{m}(b)}{\Gamma_{m}(a+b)}=B_{m}(b, a),
\end{aligned}
$$

where $\operatorname{Re}(a)>(m-1) / 2$ and $\operatorname{Re}(b)>(m-1) / 2$. Siegel [10] established the identity

$$
B_{m}(a, b)=\int_{Y>0} \operatorname{det}(Y)^{a-(m+1) / 2} \operatorname{det}\left(I_{m}+Y\right)^{-(a+b)} d Y
$$

Received by the editors June 10, 2003 and, in revised form, November 5, 2004 and June 1, 2005.

2000 Mathematics Subject Classification. Primary 33E99; Secondary 62H99.

Key words and phrases. Beta function, Dirichlet function, gamma function, Liouville integral, matrix variate, transformation.

The first and third authors were supported by the Comité para el Desarrollo de la Investigación, Universidad de Antioquia research grant no. IN387CE.

(C)2006 American Mathematical Society Reverts to public domain 28 years from publication 
which can be derived from (1.3) by using the matrix transformation $X=$ $\left(I_{m}+Y\right)^{-1} Y$. The multivariate gamma function defined above arises naturally in the probability density function (p.d.f.) of the Wishart matrix. If $W \sim W_{m}(n, \Sigma)$, $n>m-1, \Sigma>0$, then its p.d.f. is given by

$$
\left\{2^{n m / 2} \Gamma_{m}\left(\frac{n}{2}\right) \operatorname{det}(\Sigma)^{n / 2}\right\}^{-1} \operatorname{etr}\left(-\frac{\Sigma^{-1} W}{2}\right) \operatorname{det}(W)^{(n-m-1) / 2}, W>0 .
$$

Further, if $W_{1} \sim W_{m}\left(n_{1}, I_{m}\right)$ and $W_{2} \sim W_{m}\left(n_{2}, I_{m}\right)$ are independent Wishart matrices, then the p.d.f.'s of $X=\left(W_{1}+W_{2}\right)^{-1 / 2} W_{1}\left(W_{1}+W_{2}\right)^{-1 / 2}$ and $Y=$ $W_{2}^{-1 / 2} W_{1} W_{2}^{-1 / 2}$ are given by

$$
\left\{B_{m}\left(\frac{n_{1}}{2}, \frac{n_{2}}{2}\right)\right\}^{-1} \operatorname{det}(X)^{n_{1} / 2-(m+1) / 2} \operatorname{det}\left(I_{m}-X\right)^{n_{2} / 2-(m+1) / 2}, 0<X<I_{m},
$$

and

$$
\left\{B_{m}\left(\frac{n_{1}}{2}, \frac{n_{2}}{2}\right)\right\}^{-1} \operatorname{det}(Y)^{n_{1} / 2-(m+1) / 2} \operatorname{det}\left(I_{m}+Y\right)^{-\left(n_{1}+n_{2}\right) / 2}, Y>0,
$$

respectively. Thus multivariate gamma and multivariate beta functions frequently occur in the densities of random matrices (e.g., see Gupta and Nagar [3]) and hence play a pivotal role in multivariate statistical analysis.

Let $A=\left(a_{i j}\right)$ be an $m \times m$ symmetric matrix. Define $A^{(\alpha)}=\left(a_{i j}\right), 1 \leq i, j \leq \alpha$. Bellman [1] has defined a multivariate gamma function as

$$
\Gamma_{m}\left(a_{1}, \ldots, a_{m}\right)=\int_{X>0} \frac{\operatorname{etr}(-X) \operatorname{det}(X)^{a_{m}-(m+1) / 2}}{\prod_{\alpha=2}^{m} \operatorname{det}\left(X_{(\alpha)}\right)^{k_{\alpha-1}}} d X
$$

where $a_{j}=k_{m-j+1}+\cdots+k_{m}$ and $\operatorname{Re}\left(a_{j}\right)>(j-1) / 2, j=1, \ldots, m$. Using an inductive argument, Bellman [1] showed that

$$
\Gamma_{m}\left(a_{1}, \ldots, a_{m}\right)=\pi^{m(m-1) / 4} \prod_{j=1}^{m} \Gamma\left(a_{j}-\frac{j-1}{2}\right), \operatorname{Re}\left(a_{j}\right)>\frac{m-1}{2} .
$$

Olkin [7, using matrix transformation, evaluated the above integral and established similar results for the beta function. He also derived a matrix variate version of the Liouville integral.

It may be noted here that integrands in (1.1), (1.3) and (1.4) are real-valued non-negative functions of symmetric matrices and have been evaluated over the space of positive definite matrices. When these functions (or their modified forms) have a Hermitian matrix as an argument and are integrated over Hermitian positive definite matrices, complex multivariate gamma and complex multivariate beta functions are defined. These functions occur in the p.d.f. of complex Wishart and complex beta matrices and play an important role in complex multivariate statistical analysis.

In this article we study Bellman's gamma function and Olkin's beta function in the case of Hermitian positive definite matrices. We also study the Liouville integral and its generalizations to the complex case.

In Section 2 and Section 3 the generalized complex multivariate gamma and generalized complex multivariate beta functions are defined, and several properties have been studied. In Section 4, the complex multivariate Dirichlet function is studied, and the relationship between the Dirichlet function and the complex multivariate gamma function is established. Finally, in Section [5, using results of Section 2 and Section 3, we define generalized complex matrix variate gamma and 
generalized complex matrix variate beta distributions. The distributions so defined are closely related to the complex matrix variate gamma and the complex matrix variate beta distributions.

\section{Generalized COMPlex multivariate gamma FUnCtion}

In this section we will define generalized complex multivariate gamma function. We first state the following notations and results (Khatri 5, Srivastava 13]) that will be utilized in this and subsequent sections. Let $A=\left(a_{i j}\right)$ be an $m \times m$ matrix of complex numbers. Then, $A^{\prime}$ denotes the transpose of $A ; \bar{A}$ denotes conjugate of $A ; A^{H}$ denotes conjugate transpose of $A ; \operatorname{tr}(A)=a_{11}+\cdots+a_{m m}$; $\operatorname{etr}(A)=\exp (\operatorname{tr}(A)) ; \operatorname{det}(A)=$ determinant of $A ; A=A^{H}>0$ means that $A$ is Hermitian positive definite and $A^{1 / 2}$ denotes the unique Hermitian positive definite square root of $A=A^{H}>0$. The submatrices $A^{(\alpha)}$ and $A_{(\alpha)}, 1 \leq \alpha \leq m$, of the matrix $A$ are defined as $A^{(\alpha)}=\left(a_{i j}\right), 1 \leq i, j \leq \alpha$, and $A_{(\alpha)}=\left(a_{i j}\right), \alpha \leq i, j \leq m$, respectively.

Lemma 2.1 (Khatri 5]). Let $Z$ and $W$ be $m \times m$ Hermitian positive definite matrices. If $Z=G W G^{H}$, where $G(m \times m)$ is a complex nonsingular matrix, then $J(Z \rightarrow W)=\operatorname{det}\left(G G^{H}\right)^{m}$.

Lemma 2.2 (Goodman [2]). Let $W$ be a Hermitian positive definite matrix and $W=T T^{H}$, where $T$ is an $m \times m$ complex triangular matrix with positive diagonal elements. Then, (i) $J(W \rightarrow T)=2^{m} \prod_{i=1}^{m} t_{i i}^{2(m-i)+1}$ if $T$ is lower triangular, and (ii) $J(W \rightarrow T)=2^{m} \prod_{i=1}^{m} t_{i i}^{2 i-1}$ if $T$ is upper triangular.

Lemma 2.3 (Khatri [5]). Let $X=\left(x_{i j}\right), Y=\left(y_{i j}\right)$ and $T=\left(t_{i j}\right)$ be $m \times m$ complex triangular matrices with positive diagonal elements and $Y=T X$. If $T$ and $X$ are lower triangular, then $J(Y \rightarrow T)=\prod_{i=1}^{m} x_{i i}^{2(m-i)+1}$ and $J(Y \rightarrow X)=\prod_{i=1}^{m} t_{i i}^{2 i-1}$. Further, if $T$ and $X$ are upper triangular, then $J(Y \rightarrow T)=\prod_{i=1}^{m} x_{i i}^{2 i-1}$ and $J(Y \rightarrow X)=\prod_{i=1}^{m} t_{i i}^{2(m-i)+1}$.

Lemma 2.4. Let $W$ and $A$ be $m \times m$ Hermitian positive definite matrices and $W=T A T^{H}$, where $T$ is an $m \times m$ complex triangular matrix with positive diagonal elements. Then, (i) $J(W \rightarrow T)=2^{m} \operatorname{det}(A) \prod_{i=1}^{m-1} \operatorname{det}\left(A^{(i)}\right)^{2} \prod_{i=1}^{m} t_{i i}^{2(m-i)+1}$ if $T$ is lower triangular and (ii) $J(W \rightarrow T)=2^{m} \operatorname{det}(A) \prod_{i=2}^{m} \operatorname{det}\left(A_{(i)}\right)^{2} \prod_{i=1}^{m} t_{i i}^{2 i-1}$ if $T$ is upper triangular.

Proof. Write $A=X X^{H}$, where $X$ is a lower triangular matrix with positive diagonal elements, and let $Y=T X$. Then, using Lemma 2.2 and Lemma 2.3. $J(W \rightarrow T)=J(W \rightarrow Y) J(Y \rightarrow T)=\left(2^{m} \prod_{i=1}^{m} y_{i i}^{2(m-i)+1}\right)\left(\prod_{i=1}^{m} x_{i i}^{2(m-i)+1}\right)=$ $2^{m} \prod_{i=1}^{m}\left\{t_{i i}^{2(m-i)+1} x_{i i}^{4(m-i)+2}\right\}$. The result follows by noting that $\prod_{i=1}^{m} x_{i i}^{4(m-i)+2}=$ $\operatorname{det}(A) \prod_{i=1}^{m-1} \operatorname{det}\left(A^{(i)}\right)^{2}$. The proof of (ii) is similar.

The complex multivariate gamma function is defined by

$$
\tilde{\Gamma}_{m}(a)=\int_{X=X^{H}>0} \operatorname{etr}(-X) \operatorname{det}(X)^{a-m} d X
$$


where the integral is evaluated over $m \times m$ Hermitian positive definite matrices. Evaluation of the above integral yields

$$
\tilde{\Gamma}_{m}(a)=\pi^{m(m-1) / 2} \prod_{j=1}^{m} \Gamma(a-j+1), \operatorname{Re}(a)>m-1 .
$$

The complex multivariate beta function, denoted by $\tilde{B}_{m}(a, b)$, is defined by

$$
\tilde{B}_{m}(a, b)=\int_{0<X=X^{H}<I_{m}} \operatorname{det}(X)^{a-m} \operatorname{det}\left(I_{m}-X\right)^{b-m} d X
$$

where $\operatorname{Re}(a)>m-1$ and $\operatorname{Re}(b)>m-1$. The complex multivariate beta function $\tilde{B}_{m}(a, b)$ can be expressed in terms of complex multivariate gamma functions as

$$
\tilde{B}_{m}(a, b)=\frac{\tilde{\Gamma}_{m}(a) \tilde{\Gamma}_{m}(b)}{\tilde{\Gamma}_{m}(a+b)}=\tilde{B}_{m}(b, a)
$$

Now we generalize the complex multivariate gamma function as follows.

Definition 2.5. The generalized complex multivariate gamma function, denoted by $\tilde{\Gamma}_{m}^{*}\left(a_{1}, \ldots, a_{m}\right)$, is defined as

$$
\tilde{\Gamma}_{m}^{*}\left(a_{1}, \ldots, a_{m}\right)=\int_{X=X^{H}>0} \frac{\operatorname{etr}(-X) \operatorname{det}(X)^{a_{m}-m}}{\prod_{\alpha=2}^{m} \operatorname{det}\left(X_{(\alpha)}\right)^{k_{\alpha-1}}} d X
$$

where $a_{j}=k_{m-j+1}+\cdots+k_{m}$ and $\operatorname{Re}\left(a_{j}\right)>j-1, j=1, \ldots, m$.

Theorem 2.6. For $\operatorname{Re}\left(a_{j}\right)>j-1, j=1, \ldots, m$,

$$
\tilde{\Gamma}_{m}^{*}\left(a_{1}, \ldots, a_{m}\right)=\pi^{m(m-1) / 2} \prod_{j=1}^{m} \Gamma\left(a_{j}-j+1\right) .
$$

Proof. Let $X=T T^{H}$, where $T$ is an upper triangular matrix with positive diagonal elements, and partition $X$ and $T$ as

$$
X=\left(\begin{array}{ll}
X_{11} & X_{12} \\
X_{21} & X_{22}
\end{array}\right), T=\left(\begin{array}{cc}
T_{11} & T_{12} \\
0 & T_{22}
\end{array}\right)
$$

where $X_{11}$ and $T_{11}$ are $(\alpha-1) \times(\alpha-1)$ matrices. Now it is easy to see that $X_{(\alpha)}=T_{22} T_{22}^{H}, \operatorname{det}\left(X_{(\alpha)}\right)=\prod_{i=\alpha}^{m} t_{i i}^{2}, \operatorname{det}(X)=\prod_{i=1}^{m} t_{i i}^{2}$ and $\operatorname{tr}(X)=\operatorname{tr}\left(T T^{H}\right)=$ $\sum_{i<j}^{m}\left(t_{1 i j}^{2}+t_{2 i j}^{2}\right)+\sum_{i=1}^{m} t_{i i}^{2}$, where $t_{i j}=t_{1 i j}+\sqrt{-1} t_{2 i j}, 1 \leq i<j \leq m$. From 
Lemma 2.2 we have $J(X \rightarrow T)=2^{m} \prod_{i=1}^{m} t_{i i}^{2 i-1}$. Hence we can write (2.5) as

$$
\begin{aligned}
\tilde{\Gamma}_{m}^{*}\left(a_{1}, \ldots, a_{m}\right)= & 2^{m} \int_{\substack{\infty<t_{i j j}, t_{2 i j}<\infty \\
t_{i i}>0}} \exp \left[-\sum_{i<j}^{m}\left(t_{1 i j}^{2}+t_{2 i j}^{2}\right)-\sum_{i=1}^{m} t_{i i}^{2}\right] \\
& \times \prod_{i=1}^{m}\left(t_{i i}^{2}\right)^{a_{m}-m+i-1 / 2} \prod_{\alpha=2}^{m} \prod_{i=\alpha}^{m}\left(t_{i i}^{2}\right)^{-k_{\alpha-1}} \prod_{i \leq j}^{m} d t_{i j} \\
= & {\left[\prod_{i=1}^{m} 2 \int_{t_{i i}>0}\left(t_{i i}^{2}\right)^{a_{m-i+1}-m+i-1 / 2} \exp \left(-t_{i i}^{2}\right) d t_{i i}\right] } \\
& \times\left[\prod_{i<j}^{m} \int_{-\infty}^{\infty} \exp \left(-t_{1 i j}^{2}\right) d t_{1 i j}\right]\left[\prod_{i<j}^{m} \int_{-\infty}^{\infty} \exp \left(-t_{2 i j}^{2}\right) d t_{2 i j}\right] \\
= & {\left[\prod_{i=1}^{m} \int_{0}^{\infty} y_{i}^{a_{m-i+1}-m+i-1} \exp \left(-y_{i}\right) d y_{i}\right] } \\
& \times\left[\int_{-\infty}^{\infty} \exp \left(-x^{2}\right) d x\right]^{m(m-1)} .
\end{aligned}
$$

Finally, using the results $\int_{0}^{\infty} \exp \left(-y_{i}\right) y_{i}^{a_{m-i+1}-m+i-1} d y_{i}=\Gamma\left(a_{m-i+1}-m+i\right)$ and $\int_{-\infty}^{\infty} \exp \left(-x^{2}\right) d x=\sqrt{\pi}$ gives the desired result, completing the proof.

Let $y_{i j}=x_{m-i+1, m-j+1}, 1 \leq i, j \leq m, k_{i}=n_{m-i+1}$ and $b_{i}=n_{1}+\cdots+$ $n_{i}, 1 \leq i \leq m$. Then $\operatorname{tr}(X)=\operatorname{tr}(Y), \operatorname{det}(X)=\operatorname{det}(Y), \prod_{\alpha=2}^{m} \operatorname{det}\left(X_{(\alpha)}\right)^{k_{\alpha-1}}=$ $\prod_{\alpha=1}^{m-1} \operatorname{det}\left(Y^{(\alpha)}\right)^{n_{\alpha+1}}$ and $J\left(x_{i j} \rightarrow y_{m-i+1, m-j+1}\right)=1,1 \leq i, j \leq m$. Making these substitutions, the integral in (2.5) reduces to

$$
\int_{Y=Y^{H}>0} \frac{\operatorname{etr}(-Y) \operatorname{det}(Y)^{b_{m}-m}}{\prod_{\alpha=1}^{m-1} \operatorname{det}\left(Y^{(\alpha)}\right)^{n_{\alpha+1}}} d Y .
$$

Thus, using the above integral we can also define generalized complex multivariate gamma function as follows.

Definition 2.7. The generalized complex multivariate gamma function, denoted by $\tilde{\Gamma}_{m}^{*}\left(b_{1}, \ldots, b_{m}\right)$, is defined as

$$
\tilde{\Gamma}_{m}^{*}\left(b_{1}, \ldots, b_{m}\right)=\int_{Y=Y^{H}>0} \frac{\operatorname{etr}(-Y) \operatorname{det}(Y)^{b_{m}-m}}{\prod_{\alpha=1}^{m-1} \operatorname{det}\left(Y^{(\alpha)}\right)^{n_{\alpha+1}}} d Y,
$$

where $b_{j}=n_{1}+\cdots+n_{j}$ and $\operatorname{Re}\left(b_{j}\right)>j-1, j=1, \ldots, m$.

Theorem 2.8. For $\operatorname{Re}\left(a_{j}\right)>j-1, j=1, \ldots, m$, and $B=B^{H}>0$,

$$
\int_{X=X^{H}>0} \frac{\operatorname{etr}(-B X) \operatorname{det}(X)^{a_{m}-m}}{\prod_{\alpha=2}^{m} \operatorname{det}\left(X_{(\alpha)}\right)^{k_{\alpha-1}}} d X=\frac{\tilde{\Gamma}_{m}^{*}\left(a_{1}, \ldots, a_{m}\right)}{\prod_{\alpha=1}^{m} \operatorname{det}\left(B^{(\alpha)}\right)^{k_{\alpha}}},
$$

where $a_{j}=k_{m-j+1}+\cdots+k_{m}$.

Proof. Since $B=B^{H}>0$, let $B=C C^{H}$, where $C=\left(c_{i j}\right)$ is a lower triangular matrix. Substitute $\Lambda=C^{H} X C$; then $\operatorname{tr}(B X)=\operatorname{tr}\left(C C^{H} X\right)=\operatorname{tr}\left(C^{H} X C\right)=\operatorname{tr}(\Lambda)$, and $J(X \rightarrow \Lambda)=\operatorname{det}(C)^{-2 m}=\operatorname{det}(B)^{-m}$. Now partition $C, X$ and $\Lambda$ as

$$
C=\left(\begin{array}{cc}
C_{11} & 0 \\
C_{21} & C_{22}
\end{array}\right), X=\left(\begin{array}{cc}
X_{11} & X_{12} \\
X_{21} & X_{22}
\end{array}\right), \Lambda=\left(\begin{array}{cc}
\Lambda_{11} & \Lambda_{12} \\
\Lambda_{21} & \Lambda_{22}
\end{array}\right),
$$


where $C_{11}, X_{11}$ and $\Lambda_{11}$ are $(\alpha-1) \times(\alpha-1)$ matrices. Then, $\Lambda_{22}=\Lambda_{(\alpha)}=$ $C_{22}^{H} X_{22} C_{22}$, and $\operatorname{det}\left(\Lambda_{(\alpha)}\right)=\operatorname{det}\left(C_{22}^{H} X_{22} C_{22}\right)=\operatorname{det}\left(X_{22}\right) \prod_{i=\alpha}^{m} c_{i i}^{2}=\operatorname{det}\left(X_{(\alpha)}\right)$ $\prod_{i=\alpha}^{m} c_{i i}^{2}$. Thus the left hand side of (2.8) reduces to

$$
\begin{array}{r}
\frac{\operatorname{det}(B)^{-a_{m}}}{\prod_{\alpha=2}^{m} \prod_{i=\alpha}^{m}\left(c_{i i}^{-2}\right)^{k_{\alpha-1}}} \int_{\Lambda=\Lambda^{H}>0} \frac{\operatorname{det}(\Lambda)^{a_{m}-m} \operatorname{etr}(-\Lambda)}{\prod_{\alpha=2}^{m} \operatorname{det}\left(\Lambda_{(\alpha)}\right)^{k_{\alpha-1}}} d \Lambda \\
=\frac{\tilde{\Gamma}_{m}^{*}\left(a_{1}, \ldots, a_{m}\right) \operatorname{det}(B)^{-a_{m}}}{\prod_{\alpha=2}^{m} \prod_{i=\alpha}^{m}\left(c_{i i}^{-2}\right)^{k_{\alpha-1}}} .
\end{array}
$$

Now the result follows by noting that $\prod_{i=\alpha}^{m} c_{i i}^{2}=\operatorname{det}(B) / \operatorname{det}\left(B^{(\alpha-1)}\right)$.

Theorem 2.9. For $\operatorname{Re}\left(b_{j}\right)>j-1, j=1, \ldots, m$, and $B=B^{H}>0$,

$$
\int_{X=X^{H}>0} \frac{\operatorname{etr}(-B X) \operatorname{det}(X)^{b_{m}-m}}{\prod_{\alpha=1}^{m-1} \operatorname{det}\left(X^{(\alpha)}\right)^{n_{\alpha+1}}} d X=\frac{\tilde{\Gamma}_{m}^{*}\left(b_{1}, \ldots, b_{m}\right)}{\prod_{\alpha=1}^{m} \operatorname{det}\left(B_{(\alpha)}\right)^{n_{\alpha}}}
$$

where $b_{j}=n_{1}+\cdots+n_{j}$.

Proof. Similar to the proof of Theorem 2.8 .

Theorem 2.10. For $\operatorname{Re}\left(a_{j}\right)>j-1, j=1, \ldots, m$,

$$
\int_{X=X^{H}>0} \frac{\operatorname{det}(X)^{a_{m}-m} \operatorname{etr}(-X)(\operatorname{tr} X)^{r}}{\prod_{\alpha=2}^{m} \operatorname{det}\left(X_{(\alpha)}\right)^{k_{\alpha-1}}} d X=\left(\sum_{j=1}^{m} a_{j}\right)_{r} \tilde{\Gamma}_{m}^{*}\left(a_{1}, \ldots, a_{m}\right)
$$

where $a_{j}=k_{m-j+1}+\cdots+k_{m}$ with $(a)_{0}=1$ and $(a)_{r}=a(a+1) \cdots(a+r-1)=$ $(a)_{r-1}(a+r-1), r=1,2, \ldots$. Further, for $\operatorname{Re}\left(b_{j}\right)>j-1, j=1, \ldots, m$,

$$
\int_{X=X^{H}>0} \frac{\operatorname{det}(X)^{b_{m}-m} \operatorname{etr}(-X)(\operatorname{tr} X)^{r}}{\prod_{\alpha=1}^{m-1} \operatorname{det}\left(X^{(\alpha)}\right)^{n_{\alpha+1}}} d X=\left(\sum_{j=1}^{m} b_{j}\right)_{r} \tilde{\Gamma}_{m}^{*}\left(b_{1}, \ldots, b_{m}\right),
$$

where $b_{j}=n_{1}+\cdots+n_{j}$.

Proof. Setting $B=t I_{m}$ in (2.8) and differentiating the resulting identity $r$ times with respect to $t$, we obtain

$$
\begin{aligned}
& \int_{X=X^{H}>0} \frac{\exp [-t \operatorname{tr}(X)] \operatorname{det}(X)^{a_{m}-m}(\operatorname{tr} X)^{r}}{\prod_{\alpha=2}^{m} \operatorname{det}\left(X_{(\alpha)}\right)^{k_{\alpha}-1}} d X \\
& =\left(\sum_{j=1}^{m} a_{j}\right)_{r} \tilde{\Gamma}_{m}^{*}\left(a_{1}, \ldots, a_{m}\right) t^{-\left(\sum_{\alpha=1}^{m} \alpha k_{\alpha}+r\right)} .
\end{aligned}
$$

Now, substituting $t=1$ above we get the desired result. The proof of the second part follows similar steps.

\section{Generalized complex multivariate Beta function}

The generalized multivariate beta function is defined as follows.

Definition 3.1. The generalized complex multivariate beta function, denoted by $\tilde{B}_{m}^{*}\left(a_{1}, \ldots, a_{m} ; b_{1}, \ldots, b_{m}\right)$, is defined by

$$
\begin{aligned}
& \tilde{B}_{m}^{*}\left(a_{1}, \ldots, a_{m} ; b_{1}, \ldots, b_{m}\right) \\
& =\int_{0<X=X^{H}<I_{m}} \frac{\operatorname{det}(X)^{a_{m}-m} \operatorname{det}\left(I_{m}-X\right)^{b_{m}-m} d X}{\prod_{\alpha=2}^{m}\left\{\operatorname{det}\left(X_{(\alpha)}\right)^{k_{\alpha-1}} \operatorname{det}\left(\left(I_{m}-X\right)_{(\alpha)}\right)^{n_{\alpha-1}}\right\}},
\end{aligned}
$$

where $a_{j}=\sum_{i=m-j+1}^{m} k_{i}, b_{j}=\sum_{i=m-j+1}^{m} n_{i}, \operatorname{Re}\left(a_{j}\right)>j-1$, and $\operatorname{Re}\left(b_{j}\right)>j-$ $1, j=1, \ldots, m$. 
Theorem 3.2. For $\operatorname{Re}\left(a_{j}\right)>j-1$, and $\operatorname{Re}\left(b_{j}\right)>j-1, j=1, \ldots, m$,

$$
\tilde{B}_{m}^{*}\left(a_{1}, \ldots, a_{m} ; b_{1}, \ldots, b_{m}\right)=\frac{\tilde{\Gamma}_{m}^{*}\left(a_{1}, \ldots, a_{m}\right) \tilde{\Gamma}_{m}^{*}\left(b_{1}, \ldots, b_{m}\right)}{\tilde{\Gamma}_{m}^{*}\left(a_{1}+b_{1}, \ldots, a_{m}+b_{m}\right)} .
$$

Proof. We have, by Definition 2.5

$$
\begin{aligned}
& \tilde{\Gamma}_{m}^{*}\left(a_{1}, \ldots, a_{m}\right) \tilde{\Gamma}_{m}^{*}\left(b_{1}, \ldots, b_{m}\right) \\
& \quad=\int_{X=X^{H}>0} \int_{Y=Y^{H}>0} \frac{\operatorname{etr}(-X-Y) \operatorname{det}(X)^{a_{m}-m} \operatorname{det}(Y)^{b_{m}-m}}{\prod_{\alpha=2}^{m}\left\{\operatorname{det}\left(X_{(\alpha)}\right)^{k_{\alpha-1}} \operatorname{det}\left(Y_{(\alpha)}\right)^{n_{\alpha-1}}\right\}} d X d Y,
\end{aligned}
$$

where $a_{j}=\sum_{i=m-j+1}^{m} k_{i}, b_{j}=\sum_{i=m-j+1}^{m} n_{i}, \operatorname{Re}\left(a_{j}\right)>j-1$, and $\operatorname{Re}\left(b_{j}\right)>$ $j-1, j=1, \ldots, m$. Now, let $X+Y=T T^{H}$, where $T$ is an upper triangular matrix with positive diagonal elements and $W=T^{-1} X\left(T^{H}\right)^{-1}$. Then $X=$ $T W T^{H}, Y=T\left(I_{m}-W\right) T^{H}, \operatorname{det}\left(Y_{(\alpha)}\right)=\operatorname{det}\left(\left(I_{m}-W\right)_{(\alpha)}\right) \prod_{i=\alpha}^{m} t_{i i}^{2}, \operatorname{det}\left(X_{(\alpha)}\right)=$ $\operatorname{det}\left(W_{(\alpha)}\right) \prod_{i=\alpha}^{m} t_{i i}^{2}$, and $\operatorname{tr}(X+Y)=\operatorname{tr}\left(T T^{H}\right)=\sum_{i<j}^{m}\left(t_{1 i j}^{2}+t_{2 i j}^{2}\right)+\sum_{i=1}^{m} t_{i i}^{2}$, where $t_{i j}=t_{1 i j}+\sqrt{-1} t_{2 i j}, 1 \leq i<j \leq m$. The Jacobian of transformation from Lemma 2.1 and Lemma 2.2 is given by $J(X, Y \rightarrow W, T)=2^{m} \prod_{i=1}^{m} t_{i i}^{2(m+i)-1}$. Hence (3.2) can be written as

$$
\begin{aligned}
& \tilde{\Gamma}_{m}^{*}\left(a_{1}, \ldots, a_{m}\right) \tilde{\Gamma}_{m}^{*}\left(b_{1}, \ldots, b_{m}\right) \\
& =\int_{0<W=W^{H}<I_{m}} \frac{\operatorname{det}(W)^{a_{m}-m} \operatorname{det}\left(I_{m}-W\right)^{b_{m}-m}}{\prod_{\alpha=2}^{m}\left\{\operatorname{det}\left(W_{(\alpha)}\right)^{k_{\alpha}-1} \operatorname{det}\left(\left(I_{m}-W\right)_{(\alpha)}\right)^{n_{\alpha-1}}\right\}} d W \\
& \quad \times 2^{m} \int_{\substack{\sum_{1} \\
t_{1 i}, t_{2 i j}<0}} \exp \left[-\sum_{i<j}^{m}\left(t_{1 i j}^{2}+t_{2 i j}^{2}\right)-\sum_{i=1}^{m} t_{i i}^{2}\right] \\
& \prod_{i=1}^{m}\left(t_{i i}^{2}\right)^{a_{m}+b_{m}-m+i-1 / 2} \prod_{\alpha=1}^{m-1} \prod_{i=1}^{\alpha}\left(t_{i i}^{2}\right)^{-\left(k_{\alpha-1}+n_{\alpha-1}\right)} \prod_{i \leq j}^{m} d t_{i j} \\
& =\tilde{B}_{m}^{*}\left(a_{1}, \ldots, a_{m} ; b_{1}, \ldots, b_{m}\right) \tilde{\Gamma}_{m}^{*}\left(a_{1}+b_{1}, \ldots, a_{m}+b_{m}\right) .
\end{aligned}
$$

The last equality follows from Definition 3.1 and Theorem 2.6

Theorem 3.3. For $\operatorname{Re}\left(a_{j}\right)>j-1$, and $\operatorname{Re}\left(b_{j}\right)>j-1, j=1, \ldots, m$, we have

$$
\begin{aligned}
& \int_{0<X=X^{H}<I_{m}} \frac{\operatorname{det}(X)^{a_{m}-m} \operatorname{det}\left(I_{m}-X\right)^{b_{m}-m}}{\prod_{\alpha=1}^{m-1}\left\{\operatorname{det}\left(X^{(\alpha)}\right)^{k_{\alpha+1}} \operatorname{det}\left(I_{\alpha}-X^{(\alpha)}\right)^{n_{\alpha+1}}\right\}} d X \\
& =\tilde{B}_{m}^{*}\left(a_{1}, \ldots, a_{m} ; b_{1}, \ldots, b_{m}\right),
\end{aligned}
$$

where $a_{j}=\sum_{i=1}^{j} k_{i}, b_{j}=\sum_{i=1}^{j} n_{i}$.

Proof. Similar to the proof of Theorem 3.2

\section{LiOUVILLE-DiRICHLET INTEGRAL}

In this section we study multivariate Dirichlet integral, Liouville integral and its generalizations (Sivazlian [11, 12, Klamkin [6, Olkin [7, 8]) in the complex case. 
Definition 4.1. The complex multivariate Dirichlet function is denoted by $\tilde{B}_{m}\left(a_{1}, \ldots, a_{r} ; b\right)$ and is defined by

$$
\tilde{B}_{m}\left(a_{1}, \ldots, a_{r} ; b\right)=\int_{\substack{0<\sum_{i=1}^{r}=1 \\ X_{i}=X_{i}^{H}>0, i=1, \ldots, r}} \ldots \prod_{i=1}^{r} \operatorname{det}\left(X_{i}\right)^{a_{i}-m} \operatorname{det}\left(I_{m}-\sum_{i=1}^{r} X_{i}\right)^{b-m} \prod_{i=1}^{r} d X_{i},
$$

where $\operatorname{Re}\left(a_{i}\right)>m-1, i=1, \ldots, r$, and $\operatorname{Re}(b)>m-1$.

The relation between the complex multivariate Dirichlet function and the complex multivariate gamma function is given in the following theorem.

Theorem 4.2. For $\operatorname{Re}\left(a_{i}\right)>m-1, i=1, \ldots, r$, and $\operatorname{Re}(b)>m-1$,

$$
\tilde{B}_{m}\left(a_{1}, \ldots, a_{r} ; b\right)=\frac{\tilde{\Gamma}_{m}(b) \prod_{i=1}^{r} \tilde{\Gamma}_{m}\left(a_{i}\right)}{\tilde{\Gamma}_{m}(a+b)},
$$

where $a=\sum_{i=1}^{r} a_{i}$.

Proof. Consider the integral

$$
\phi(X)=\int_{\substack{\sum_{i}^{r}=1 \\ X_{i}=X_{i}^{H}>0, i=1, \ldots, r}} \ldots \prod_{i=1}^{r} \operatorname{det}\left(X_{i}\right)^{a_{i}-m} \operatorname{det}\left(I_{m}-\sum_{i=1}^{r} X_{i}\right)^{b-m} \prod_{i=1}^{r} d X_{i} .
$$

Substituting $\sum_{i=1}^{r} X_{i}=X, W_{i}=X^{-1 / 2} X_{i} X^{-1 / 2}, i=1, \ldots, r-1$, in (4.2) with the Jacobian

$$
J\left(X_{1}, \ldots, X_{r-1}, X_{r} \rightarrow W_{1}, \ldots, W_{r-1}, X\right)=\operatorname{det}(X)^{(r-1) m},
$$

we get

$$
\begin{aligned}
& \times \underset{\substack{0<\sum_{i=1}^{r-1} W_{i}<I_{m} \\
W_{i}=W_{i}^{H}>0, i=1, \ldots, r-1}}{ } \prod_{i=1}^{r-1} \operatorname{det}\left(W_{i}\right)^{a_{i}-m} \operatorname{det}\left(I_{m}-\sum_{i=1}^{r-1} W_{i}\right)^{a_{r}-m} \prod_{i=1}^{r-1} d W_{i} \\
= & \operatorname{det}(X)^{a-m} \operatorname{det}\left(I_{m}-X\right)^{b-m} \tilde{B}_{m}\left(a_{1}, \ldots, a_{r-1} ; a_{r}\right) .
\end{aligned}
$$

Now from (4.3) and (2.3) we can write

$$
\begin{aligned}
\tilde{B}_{m}\left(a_{1}, \ldots, a_{r} ; b\right)= & \int_{0<X=X^{H}<I_{m}} \phi(X) d X \\
= & \tilde{B}_{m}\left(a_{1}, \ldots, a_{r-1} ; a_{r}\right) \\
& \times \int_{0<X=X^{H}<I_{m}} \operatorname{det}(X)^{a-m} \operatorname{det}\left(I_{m}-X\right)^{b-m} d X \\
= & \tilde{B}_{m}\left(a_{1}, \ldots, a_{r-1} ; a_{r}\right) \tilde{B}_{m}(a, b) .
\end{aligned}
$$

From the recurrence relation (4.4) we get

$$
\tilde{B}_{m}\left(a_{1}, \ldots, a_{r} ; b\right)=\tilde{B}_{m}\left(\sum_{i=1}^{r} a_{i}, b\right) \tilde{B}_{m}\left(\sum_{i=1}^{r-1} a_{i}, a_{r}\right) \cdots \tilde{B}_{m}\left(a_{1}, a_{2}\right) .
$$

Substituting for the complex multivariate beta functions in (4.5) and simplifying gives the result. 
The following result is the complex matrix variate analog of Liouville's extension of the Dirichlet integral.

Theorem 4.3. Let $f(X)$ be a continuous scalar function of an $m \times m$ Hermitian matrix $X$ and let $B$ be a Hermitian positive definite matrix of order $m$. Then, for $\operatorname{Re}\left(a_{i}\right)>m-1, i=1, \ldots, r$, and $\sum_{i=1}^{r} a_{i}=a$,

$$
\begin{aligned}
& \int_{\substack{0 \\
0<\sum_{i=1}^{r} \\
X_{i}=X_{i}^{H}>0, i=1, \ldots, r}} \prod_{i=1}^{r} \operatorname{det}\left(X_{i}\right)^{a_{i}-m} f\left(\sum_{i=1}^{r} X_{i}\right) \prod_{i=1}^{r} d X_{i} \\
= & \tilde{B}_{m}\left(a_{1}, \ldots, a_{r-1} ; a_{r}\right) \int_{0<X=X^{H}<B} \operatorname{det}(X)^{a-m} f(X) d X \\
= & \frac{\prod_{i=1}^{r} \tilde{\Gamma}_{m}\left(a_{i}\right)}{\tilde{\Gamma}_{m}\left(\sum_{i=1}^{r} a_{i}\right)} \int_{0<X=X^{H}<B} \operatorname{det}(X)^{a-m} f(X) d X .
\end{aligned}
$$

Proof. Making the same transformation as in Theorem 4.2, the above left-hand side integral becomes

$$
\begin{gathered}
\int_{\substack{r-1 \\
\sum_{i=1}^{r-1} W_{i}<I_{m} \\
W_{i}=W_{i}^{H}>0, i=1, \ldots, r-1}} \prod_{i=1}^{r-1} \operatorname{det}\left(W_{i}\right)^{a_{i}-m}\left(I_{m}-\sum_{i=1}^{r-1} W_{i}\right)^{a_{r}-m} \prod_{i=1}^{r-1} d W_{i} \\
\times \int_{0<X=X^{H}<B} \operatorname{det}(X)^{a-m} f(X) d X .
\end{gathered}
$$

The desired result now follows from Definition 4.1 and (4.1).

The next two theorems give generalizations of the above theorem. We will give proof of Theorem 4.4. The proof of Theorem 4.5 can be constructed along similar lines.

Theorem 4.4. Let $f(X)$ be a continuous scalar function of an $m \times m$ Hermitian matrix $X$ and let $B$ be a Hermitian positive definite matrix of order $m$. Then

$$
\begin{aligned}
& \int_{\substack{0<\sum_{i=1}^{r+s} X_{i}<B \\
X_{i}=X_{i}^{H}>0, i=1, \ldots, r+s}} \prod_{i=1}^{r+s} \operatorname{det}\left(X_{i}\right)^{a_{i}-m} f\left(\sum_{i=1}^{r} X_{i}\right) \prod_{i=1}^{r+s} d X_{i} \\
= & \tilde{B}_{m}\left(a_{1}, \ldots, a_{r-1} ; a_{r}\right) \tilde{B}_{m}\left(a_{r+1}, \ldots, a_{r+s-1} ; a_{r+s}\right) \tilde{B}_{m}\left(a^{(2)}, m\right) \\
& \times \int_{0<X=X^{H}<B} \operatorname{det}(X)^{a^{(1)}-m} \operatorname{det}(B-X)^{a^{(2)}} f(X) d X,
\end{aligned}
$$

where $a^{(1)}=\sum_{i=1}^{r} a_{i}, a^{(2)}=\sum_{i=r+1}^{r+s} a_{i}$ and $a_{i}>m-1, i=1, \ldots, r+s$.

Proof. Transforming $\sum_{i=1}^{r} X_{i}=X, \sum_{i=r+1}^{r+s} X_{i}=Y, W_{i}=X^{-1 / 2} X_{i} X^{-1 / 2}, i=$ $1, \ldots, r-1, W_{i}=Y^{-1 / 2} X_{i} Y^{-1 / 2}, i=r+1, \ldots, r+s-1$, with the Jacobian

$$
\begin{aligned}
& J\left(X_{1}, \ldots, X_{r+s} \rightarrow W_{1}, \ldots, W_{r-1}, X, W_{r+1}, \ldots, W_{r+s-1}, Y\right) \\
& =\operatorname{det}(X)^{(r-1) m} \operatorname{det}(Y)^{(s-1) m}
\end{aligned}
$$


on the left-hand side of the above integral, we get

$$
\begin{gathered}
\int_{\substack{0<\sum_{i=1}^{r-1} W_{i}<I_{m} \\
W_{i}=W_{i}^{H}>0, i=1, \ldots, r-1}} \prod_{i=1}^{r-1} \operatorname{det}\left(W_{i}\right)^{a_{i}-m} \operatorname{det}\left(I_{m}-\sum_{i=1}^{r-1} W_{i}\right)^{a_{r}-m} \prod_{i=1}^{r-1} d W_{i} \\
\times \int_{\substack{0<\sum_{i=s-1}^{r+s-1} W_{i}<I_{m} \\
W_{i}=W_{i}^{H}>0, i=r+1, \ldots, r+s-1}} \prod_{i=r+1}^{r+s-1} \operatorname{det}\left(W_{i}\right)^{a_{i}-m} \operatorname{det}\left(I_{m}-\sum_{i=r+1}^{r+s-1} W_{i}\right)^{a_{r+s}-m} \prod_{i=r+1}^{r+s-1} d W_{i} \\
\times \int_{\substack{0<X+Y<B \\
X=X^{H}>0, Y=Y^{H}>0}} \operatorname{det}(X)^{a^{(1)}-m} \operatorname{det}(Y)^{a^{(2)}-m} f(X) d X d Y .
\end{gathered}
$$

Now, the first two integrals are readily evaluated as $\tilde{B}_{m}\left(a_{1}, \ldots, a_{r-1} ; a_{r}\right)$ and $\tilde{B}_{m}\left(a_{r+1}, \ldots, a_{r+s-1} ; a_{r+s}\right)$. Substituting $Z=(B-X)^{-1 / 2} Y(B-X)^{-1 / 2}$ with Jacobian $J(Y \rightarrow Z)=\operatorname{det}(B-X)^{m}$ in the third integral, we obtain

$$
\begin{aligned}
& \iint_{\substack{0<X+Y<B \\
X=X^{H}>0, Y=Y^{H}>0}} \operatorname{det}(X)^{a^{(1)}-m} \operatorname{det}(Y)^{a^{(2)}-m} f(X) d X d Y \\
= & \int_{0<X=X^{H}<B} \operatorname{det}(X)^{a^{(1)}-m} \operatorname{det}(B-X)^{a^{(2)}} f(X) d X \\
& \times \int_{0<Z=X^{H}<I_{m}} \operatorname{det}(Z)^{a^{(2)}-m} d Z \\
= & \tilde{B}_{m}\left(a^{(2)}, m\right) \int_{0<X=X^{H}<B} \operatorname{det}(X)^{a^{(1)}-m} \operatorname{det}(B-X)^{a^{(2)}} f(X) d X,
\end{aligned}
$$

where the last line has been obtained by using (2.3).

Theorem 4.5. Let $f$ and $g$ be continuous scalar functions of an $m \times m$ Hermitian matrix argument and let $B$ be a Hermitian positive definite matrix of order $m$. Then

$$
\begin{aligned}
& \int_{0<\sum_{i=1}^{r+s} X_{i}<B} \ldots \prod_{i=1}^{r+s} \operatorname{det}\left(X_{i}\right)^{a_{i}-m} f\left(\sum_{i=1}^{r} X_{i}\right) g\left(\sum_{i=r+1}^{r+s} X_{i}\right) \prod_{i=1}^{r+s} d X_{i} \\
& X_{i}=X_{i}^{H}>0, i=1, \ldots, r+s \\
& =\tilde{B}_{m}\left(a_{1}, \ldots, a_{r-1} ; a_{r}\right) \quad \int_{0<X+\sum^{r+s}+X_{i}<B} \operatorname{det}(X)^{a^{(1)}-m} \\
& \begin{array}{c}
0<X+\sum_{i=r+1} X_{i}<B \\
X=X^{H}>0, X_{i}=X_{i}^{H}>0, i=r+1, \ldots, r+s
\end{array} \\
& \prod_{i=r+1}^{r+s} \operatorname{det}\left(X_{i}\right)^{a_{i}-m} f(X) g\left(\sum_{i=r+1}^{r+s} X_{i}\right) d X \prod_{i=r+1}^{r+s} d X_{i} \\
& =\tilde{B}_{m}\left(a_{1}, \ldots, a_{r-1} ; a_{r}\right) \tilde{B}_{m}\left(a_{r+1}, \ldots, a_{r+s-1} ; a_{r+s}\right) \\
& \times \quad \iint_{\substack{0<X+Y<B \\
X=X^{H}>0, Y=Y^{H}>0}} \operatorname{det}(X)^{a^{(1)}-m} \operatorname{det}(Y)^{a^{(2)}-m} f(X) g(Y) d X d Y,
\end{aligned}
$$

where $a^{(1)}=\sum_{i=1}^{r} a_{i}, a^{(2)}=\sum_{i=r+1}^{r+s} a_{i}$ and $a_{i}>m-1, i=1, \ldots, r+s$. 


\section{RELATED Distributions}

In this section, using results of Section 2 and Section 3 , we define generalized complex matrix variate gamma and generalized complex matrix variate beta distributions. The distributions so defined are closely related to the complex matrix variate gamma and the complex matrix variate beta distributions. Further, we show that these distributions arise naturally in statistical distribution theory.

Definition 5.1. The generalized complex matrix variate gamma kind 1 distribution is defined by the p.d.f.

$$
\frac{\prod_{\alpha=1}^{m} \operatorname{det}\left(B^{(\alpha)}\right)^{k_{\alpha}}}{\tilde{\Gamma}_{m}^{*}\left(a_{1}, \ldots, a_{m}\right)} \frac{\operatorname{etr}(-B X) \operatorname{det}(X)^{a_{m}-m}}{\prod_{\alpha=2}^{m} \operatorname{det}\left(X_{(\alpha)}\right)^{k_{\alpha-1}}}, X=X^{H}>0,
$$

where $a_{j}>j-1, j=1, \ldots, m$, with $a_{j}=k_{m-j+1}+\cdots+k_{m}$ and $B=B^{H}>0$.

Definition 5.2. The generalized complex matrix variate gamma kind 2 distribution is defined by the p.d.f.

$$
\frac{\prod_{\alpha=1}^{m} \operatorname{det}\left(B_{(\alpha)}\right)^{-n_{\alpha}}}{\tilde{\Gamma}_{m}^{*}\left(b_{1}, \ldots, b_{m}\right)} \frac{\operatorname{etr}(-B X) \operatorname{det}(X)^{b_{m}-m}}{\prod_{\alpha=1}^{m-1} \operatorname{det}\left(X^{(\alpha)}\right)^{n_{\alpha+1}}}, X=X^{H}>0
$$

where $b_{j}>j-1, j=1, \ldots, m$, with $b_{j}=n_{1}+\cdots+n_{j}$ and $B=B^{H}>0$.

For $k_{1}=\cdots=k_{m-1}=0$ and $k_{m}=a_{m}=a$, the density in (5.1) slides to

$$
\left\{\tilde{\Gamma}_{m}(a)\right\}^{-1} \operatorname{det}(B)^{a} \operatorname{etr}(-B X) \operatorname{det}(X)^{a-m},
$$

which is a complex matrix variate gamma distribution denoted by $X \sim \mathbb{C} G_{m}(a, B)$. Similarly, by substituting $n_{1}=b_{1}=b$ and $n_{2}=\cdots=n_{m}=0$, one can see that the p.d.f. (5.2) reduces to a complex matrix variate gamma density with parameters $(b, B)$.

Definition 5.3. The generalized complex matrix variate beta kind 1 distribution is defined by

$$
\begin{aligned}
& \left\{\tilde{B}_{m}^{*}\left(a_{1}, \ldots, a_{m} ; b_{1}, \ldots, b_{m}\right)\right\}^{-1} \\
& \times \frac{\operatorname{det}(X)^{a_{m}-m} \operatorname{det}\left(I_{m}-X\right)^{b_{m}-m}}{\prod_{\alpha=2}^{m}\left\{\operatorname{det}\left(X_{(\alpha)}\right)^{k_{\alpha-1}} \operatorname{det}\left(\left(I_{m}-X\right)_{(\alpha)}\right)^{n_{\alpha-1}}\right\}},
\end{aligned}
$$

where $a_{j}=\sum_{i=m-j+1}^{m} k_{i}, b_{j}=\sum_{i=m-j+1}^{m} n_{i}, \operatorname{Re}\left(a_{j}\right)>j-1$, and $\operatorname{Re}\left(b_{j}\right)>j-$ $1, j=1, \ldots, m$.

Definition 5.4. The generalized complex matrix variate beta kind 2 distribution is defined by

$$
\begin{aligned}
& \left\{\tilde{B}_{m}^{*}\left(a_{1}, \ldots, a_{m} ; b_{1}, \ldots, b_{m}\right)\right\}^{-1} \\
& \times \frac{\operatorname{det}(X)^{a_{m}-m} \operatorname{det}\left(I_{m}-X\right)^{b_{m}-m}}{\prod_{\alpha=1}^{m-1}\left\{\operatorname{det}\left(X^{(\alpha)}\right)^{k_{\alpha+1}} \operatorname{det}\left(\left(I_{m}-X\right)^{(\alpha)}\right)^{n_{\alpha+1}}\right\}},
\end{aligned}
$$

where $a_{j}=\sum_{i=1}^{j} k_{i}, b_{j}=\sum_{i=1}^{j} n_{i}, \operatorname{Re}\left(a_{j}\right)>j-1$, and $\operatorname{Re}\left(b_{j}\right)>j-1, j=1, \ldots, m$.

Note that by specializing parameters in (5.4) and (5.5) one can obtain complex matrix variate beta density. In the next theorem we derive generalized complex matrix variate beta densities. 
Theorem 5.5. Let $A_{i} \sim \mathbb{C} G_{m}\left(\nu_{i}, I_{m}\right), i=1,2$, be independent.

(i) If $A_{2}=T T^{H}$, where $T$ is a complex lower triangular matrix with positive diagonal elements, then the distribution of $U=T^{H}\left(A_{1}+A_{2}\right)^{-1} T$ is given by

$$
\frac{\operatorname{det}(U)^{\nu_{2}-1} \operatorname{det}\left(I_{m}-U\right)^{\nu_{1}-m}}{\tilde{B}_{m}\left(\nu_{2}, \nu_{1}\right) \prod_{i=2}^{m} \operatorname{det}\left(U_{(i)}\right)^{2}}, 0<U=U^{H}<I_{m} .
$$

Further, $U$ and $A_{1}+A_{2}$ are independently distributed.

(ii) If $A_{2}=T T^{H}$, where $T$ is a complex upper triangular matrix with positive diagonal elements, then the distribution of $U=T^{H}\left(A_{1}+A_{2}\right)^{-1} T$ is given by

$$
\frac{\operatorname{det}(U)^{\nu_{2}-1} \operatorname{det}\left(I_{m}-U\right)^{\nu_{1}-m}}{\tilde{B}_{m}\left(\nu_{2}, \nu_{1}\right) \prod_{i=1}^{m-1} \operatorname{det}\left(U^{(i)}\right)^{2}}, 0<U=U^{H}<I_{m} .
$$

Further, $U$ and $A_{1}+A_{2}$ are independently distributed.

Proof. (i) The joint p.d.f. of $A_{1}$ and $A_{2}$ is

$$
\frac{\operatorname{etr}\left[-\left(A_{1}+A_{2}\right)\right] \operatorname{det}\left(A_{1}\right)^{\nu_{1}-m} \operatorname{det}\left(A_{2}\right)^{\nu_{2}-m}}{\tilde{\Gamma}_{m}\left(\nu_{1}\right) \tilde{\Gamma}_{m}\left(\nu_{2}\right)} .
$$

Transforming $A=A_{1}+A_{2}$ and $A_{2}=T T^{H}$, where $T=\left(t_{i j}\right), t_{i i}>0, t_{i j}=$ $t_{1 i j}+\sqrt{-1} t_{2 i j}, i>j$, with the Jacobian of the transformation $J\left(A_{1}, A_{2} \rightarrow A, T\right)=$ $2^{m} \prod_{i=1}^{m} t_{i i}^{2(m-i)+1}$, we get the joint p.d.f. of $A$ and $T$ as

$$
\frac{2^{m} \operatorname{etr}(-A) \operatorname{det}\left(A-T T^{H}\right)^{\nu_{1}-m} \prod_{i=1}^{m}\left(t_{i i}^{2}\right)^{\nu_{2}-i+1 / 2}}{\tilde{\Gamma}_{m}\left(\nu_{1}\right) \tilde{\Gamma}_{m}\left(\nu_{2}\right)},
$$

where $A-T T^{H}>0, t_{i i}>0$ and $-\infty<t_{1 i j}, t_{2 i j}<\infty, i>j$. Further, transforming $U=T^{H} A^{-1} T$ with the Jacobian

$$
J(T \rightarrow U)=\left[2^{m} \operatorname{det}\left(A^{-1}\right) \prod_{i=2}^{m} \operatorname{det}\left(\left(A^{-1}\right)_{(i)}\right)^{2} \prod_{i=1}^{m} t_{i i}^{2(i-1)+1}\right]^{-1},
$$

we get the joint p.d.f. of $A$ and $U$,

$$
\frac{\operatorname{etr}(-A) \operatorname{det}(A)^{\nu_{1}+\nu_{2}-m} \operatorname{det}\left(I_{m}-U\right)^{\nu_{1}-m} \operatorname{det}(U)^{\nu_{2}-1}}{\tilde{\Gamma}_{m}\left(\nu_{1}\right) \tilde{\Gamma}_{m}\left(\nu_{2}\right) \prod_{i=2}^{m} \operatorname{det}\left(U_{(i)}\right)^{2}},
$$

since $\prod_{i=1}^{m} t_{i i}^{2 i}=\prod_{i=1}^{m}\left\{\operatorname{det}\left(U_{(i)}\right) / \operatorname{det}\left(\left(A^{-1}\right)_{(i)}\right)\right\}$. From (5.8), it is easy to see that $A$, i.e., $A_{1}+A_{2}$ and $U$ are independent, $A \sim \mathbb{C} G_{m}\left(\nu_{1}+\nu_{2}, I_{m}\right)$ and the distribution of $U$ is given by (5.6).

(ii) The proof is similar to part (i).

Theorem [5.5] in the real case, was derived by Olkin and Rubin 9 .

\section{REFERENCES}

1. R. Bellman, A generalization of some integral identities due to Ingham and Siegel, Duke Math. J., 23 (1956), 571-577. MR0081921 (18:468a)

2. N. R. Goodman, Statistical analysis based on a certain multivariate complex Gaussian distribution (an introduction), Ann. Math. Statist., 34 (1963), 152-177. MR0145618 (26:3148a)

3. A. K. Gupta and D. K. Nagar, Matrix Variate Distributions, Chapman \& Hall/CRC, Boca Raton, 2000. MR:1738933 (2001d:62055)

4. A. E. Ingham, An integral which occurs in statistics, Proc. Cambridge Philos. Soc., 29 (1933), 271-276.

5. C. G. Khatri, Classical statistical analysis based on a certain multivariate complex Gaussian distribution, Ann. Math. Statist., 36 (1965), 98-114. MR0192598(33:823) 
6. M. S. Klamkin, Extensions of Dirichlet's multiple integral, SIAM J. Math. Anal., 2 (1971), 467-469. MR0286953 (44 \#4160)

7. I. Olkin, A class of integral identities with matrix argument, Duke Math. J., 26 (1959), 207213. MR0101223 (21:36)

8. I. Olkin, Matrix extensions of Liouville-Dirichlet-type integrals, Linear Algebra Appl., 28 (1979), 155-160. MR0549430 (80i:26015)

9. I. Olkin and H. Rubin, Multivariate beta distributions and independence properties of the Wishart distribution, Ann. Math. Statist, 35 (1964), 261-269. Correction Ann. Math. Statist., 37 (1966), 297. MR0160297 (28:3511)

10. Carl Ludwig Siegel, Über die analytische theorie der quadratischen formen, Ann. Math., 36 (1935), no. 3, 527-606. MR1503238

11. B. D. Sivazlian, The generalized Dirichlet's multiple integral, SIAM Rev., 11 (1969), 285-288. MR0247014 (40:283)

12. B. D. Sivazlian, A class of multiple integrals, SIAM J. Math. Anal., 2 (1971), 72-75. MR 0285680 (44:2898)

13. M. S. Srivastava, On the complex Wishart distribution, Ann. Math. Statist., 36 (1965), 313315. MR0172401(30:2620)

Departamento de Matemáticas, Universidad de Antioquia, Medellín, AA 1226, ColomBIA

E-mail address: nagar@matematicas.udea.edu.co

Department of Mathematics and Statistics, Bowling Green State University, Bowling Green, Ohio 43403-0221

E-mail address: gupta@bgnet.bgsu.edu

Departamento de Matemáticas, Universidad de Antioquia, Medellín, AA 1226, ColomBIA

E-mail address: lesanchez@matematicas.udea.edu.co 